\title{
Oligopeptide Fragmentation Viewed as Constant Neutral Loss'
}

\author{
C. Fenselau and C. M. Murphy \\ Department of Chemistry and Biochemistry, University of Maryland Baltimore County, \\ Baltimore, Maryland, USA
}

\section{R. J. Cotter}

Department of Pharmacology and Molecular Sciences, Johns Hopkins University, Baltimore, Maryland, USA

Spectra were recorded of all fragment ions formed by elimination of 28-u neutral fragments in fast atom bombardment spectra of peptides in the mass range 1000-1800 $\mathrm{a}$. This approach can provide less complex spectra than either conventionally scanned spectra or product ion scans from collisionally activated four-sector experiments, and spectra that contain information that is both overlapping and complementary to those from the other techniques. Constant neutral loss spectra may provide a reading frame for distinguishing sequence ion series in tandem or single analyzer spectra. (I Am Soc Mass Spectrom 1990, 1, 336-340)

$\mathrm{T}$ The observation of decompositions defined by constancy in the mass of the neutral fragment [1-3] has allowed mechanism-based enhancement of both signal-to-noise $(\mathrm{S} / \mathrm{N})$ ratios and specificity for characterizing targeted analytes in complex matrices. Working with tripeptides and tetrapeptides, dePauw et al. [4] suggested that the constant neutral loss (CNL) scan can extend an analogous advantage to peptide sequencing.

Peptide spectra obtained by fast atom bombardment (FAB) contain series of ions formed by cleavages in the amide bonds. Nine series have been defined to date [5]. However, usually not all series are observed in any given spectrum, and predictive correlations between sequence and series are not yet well developed. In order to extend mass spectrometric sequencing techniques to unknown peptides more routinely, techniques are needed to direct more selective fragmentation of peptides. Some of the approaches currently under development include laser ionization [6], derivatization [5], and neutralization followed by chemical reionization $[7,8]$.

In this paper we explore the possibility that constant neutral loss (CNL) scans might offer another approach to simplified and selective spectra of peptides. In this report the approach is evaluated with peptides in the mass range currently most susceptible to mass spectrometric sequencing and having a vari-

\footnotetext{
* This work was presented at the 37th ASMS Conference on Mass Spectrometry and Allied Topics, Miami Beach, Florida, 1989.

Address reprint requests to Catherine Fenselau, Department of Chemistry and Biochemistry, University of Maryland Baltimore County, Baltimore, MD 21228.
}

ety of structures. The loss of $28 \mathrm{u}$ is monitored on a double-focusing mass spectrometer using the scan law $B^{2}(1-E) / E^{2}=$ constant.

\section{Experimental}

Bradykinin, luteinizing hormone-releasing hormone (LHRH), porcine renin substrate tetradecapeptide, and thioglycerol were obtained from Sigma Chemical Co. (5t. Louis, MO). 3-Nitrobenzyl alcohol (NBA) was obtained from Aldrich Chemical Co. (Milwaukee, WI).

Constant neutral loss scans and conventional $\left(B^{2}\right)$ magnet scans were made on the first two sectors (EB) of a JEOL (Tokyo, Japan) HX110/HX110 mass spectrometer by using the JEOL FAB gun and the JEOL DA 5000 data system. The CNL scans were measured of decompositions induced by collisions in the first fieldfree region, by using helium as target gas at pressures that attenuated the protonated molecular ion by $80 \%$.

Tandem measurements were obtained by using all four sectors (EBEB) on the JEOL HX110/HX110 instrument. Product ions were scanned $(B / E)$ on MS-2, following mass selection of precursor ions by MS-1. In these experiments helium was used for collisional activation between the two mass spectrometers, at pressure sufficient to attenuate the precursor ion beam by $80 \%$. Accelerating voltage in MS- 1 was $10 \mathrm{kV}$, and the collision cell was floated at $4 \mathrm{kV}$.

For all three scanning modes, CNL, conventional magnetic, and product ion scans in MS-2 following collisional activation, cation FAB spectra of the peptides dissolved in $0.1 \%$ trifluoroacetic acid were measured from both thioglycerol and NBA matrices. Three to six scans were averaged for each spectrum. 
A computer program developed by David Heller in the Middle Atlantic Mass Spectrometry Center was used to predict fragment ions on the basis of each peptide's sequence.

\section{Results and Discussion}

Initially scans were recorded of cation spectra formed by loss of $17,18,28,45$, and $46 u$. In this study, scans of the loss of 17 and $18 \mathrm{u}$ provided similar patterns, and many matrix cluster ions were found to undergo loss of 17 and/or $18 \mathrm{u}$. With the peptides studied here, relatively few transitions were detected with loss of 44 or $46 \mathrm{u}$. The elimination of neutral fragmenls of mass 28 provided the most complete sequence series and was selected for more detailed evaluation. The selectivity provided by the CNL scans was evaluated using a set of a-series ions [5] in the CNL spectrum of LHRH.<smiles></smiles>

For this experiment, these ions were considered to be formed exclusively by loss of $\mathrm{CO}$ from b-series precursors, that is, by the loss of $2 \dot{8} \mathrm{u}$ (Scheme I). Scans were recorded for CNL 24-30 u. Similar spectra were obtained for CNL 27 and $28 \mathrm{u}$, and the a-series ions were also detected weakly in CNL $26 \mathrm{u}$ scans. These fragment ions were not detected, however, in CNL scans for $24,25,29$, or $30 \mathrm{u}$. This window suggests that CNL scans carried out with the combination of an electrostatic and a magnetic sector may detect losses of neutral fragments one or two mass units heavier than that being scanned, but not lighter. Thus some of the fragment ions recorded in CNL 28 u scans could arise from processes in which 29-u neutral fragments were eliminated.

Scans of fragment ions formed by loss of $28 \mathrm{u}$ in the decompositions of LHRH, bradykinin, and renin substrate are presented in Figures 1a, 2, and 3, respectively. The $\mathbf{S} / \mathrm{N}$ ratio is generally good; however, the contributions of signals from the matrix become increasingly noticeable below $m / z$ 400. Scans for CNL 28 $\mathbf{u}$ from the two matrices used are presented in Figure 4. Molecular ion species are detected in all the CNL spectra, presumably without formation from heavier precursors. Prominent peaks in all the spectra are annotated with the fragment ions to which they correspond, following the conventions of Johnson et al. [5].

In theory, decompositions involving loss of 28 (or 29) $u$ could include $b \rightarrow a$ and $x+1 \rightarrow y-1$, with loss of $\mathrm{CO} ; \mathrm{w} \rightarrow \mathrm{x}$ and $\mathrm{d} \rightarrow \mathrm{c}$, with loss of $\mathrm{C}_{2} \mathrm{H}_{4}$; and $v \rightarrow x$, with loss of $\mathrm{CH}_{3} \mathrm{~N}$, the latter three transformations being precluded in certain amino acid residues. When isoleucine is present (as in renin substrate), the transformations $\mathrm{z} \rightarrow \mathrm{w}$ and $\mathrm{a} \rightarrow \mathrm{d}$ can occur by loss of $\mathrm{C}_{2} \mathrm{H}_{4}$ from the side chain.

The spectra shown in Figures 1-3 and also that of substance $P$ show that a-series ions are detected with the greatest frequency, completeness, and abundance, with contributions from $y-1, x, d, w$, and $b$ ions, in decreasing order of frequency. Among these, peaks assigned as $d, w$, or b require special comment.<smiles>[R2]C(NC(=O)NC([R2])C(=O)NC([R2])C(=O)NC([R])C(=O)NC(=O)[PH2+])C(=O)O</smiles><smiles>[R]C(NC(=O)C([R2])NC(=O)O)C(=O)O</smiles><smiles>[R2]C(NC(=O)[C@H](C)NC(=O)[C@@H]([R2])C(=O)O)C(=O)O</smiles> 

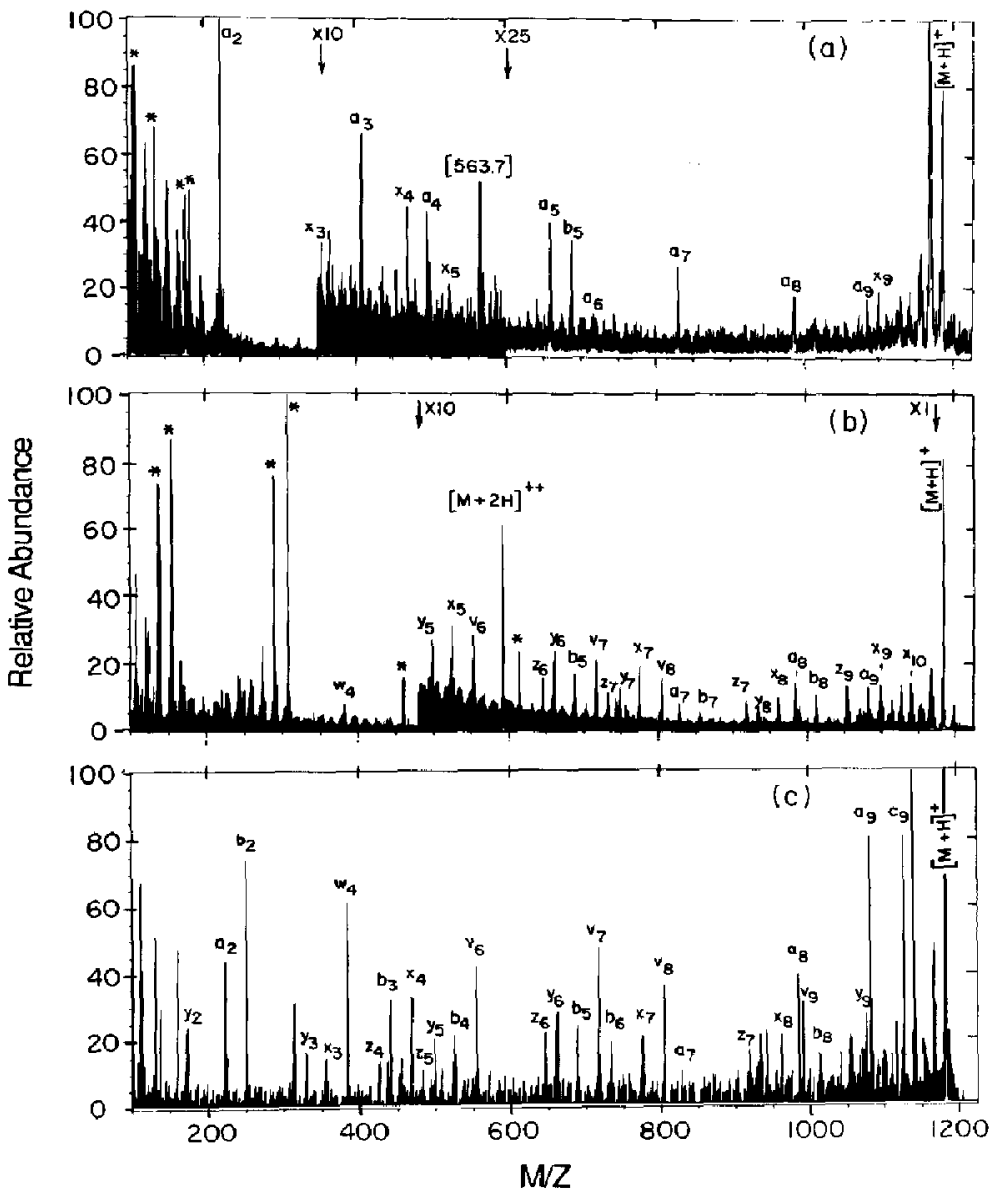

Figure 1. Spectra of LHRH (pyroGlu-HisTrp-Ser-Tyr-Gly-Leu-Arg-Pro-Gly-NH ${ }_{2}$ ), monoisotopic mass $1181.6 \mathrm{u}$, desorbed as cations by FAB. (a) Product ions formed by loss of $28 \mathrm{u}$ and analyzed by the CNL scan $\left[B^{2}(1-\right.$ $E) / E^{2}$ J. (b) Ions analyzed using a conventional magnet scan $\left(B^{2}\right)$. (c) Product ions formed by collisional activation in a four-sector experiment and analyzed by $B / E$ scan in MS-2. 3Nitrobenzyl alcohol matrix cluster ions are indicated by *
Figure 1 presents the CNL, conventional $\left(B^{2}\right)$, and product ion ( $B / E$ in MS-2) scans of LHRH. The Nterminus amino group is cyclized as an amide, and the carboxy terminus group is an amide. This peptide has strongly basic residues near both the N-terminus (histidine) and the C-terminus (arginine), and ion series are ubserved in the conventional and product ion (four-sector) spectra [9] that incorporate residues from both ends of the peptide. These $a, b, c, x, y$, and $z$ series are further overlaid by $\mathrm{v}$ and $\mathrm{w}$ ions formed by cleavages in amino acid side chains. The CNL $28 \mathrm{u}$ spectrum of LHRH contains fewer ions than the other two, and the most abundant ions are a-series ions, encompassing the series $a_{2}-a_{9}$ for this decapeptide. The

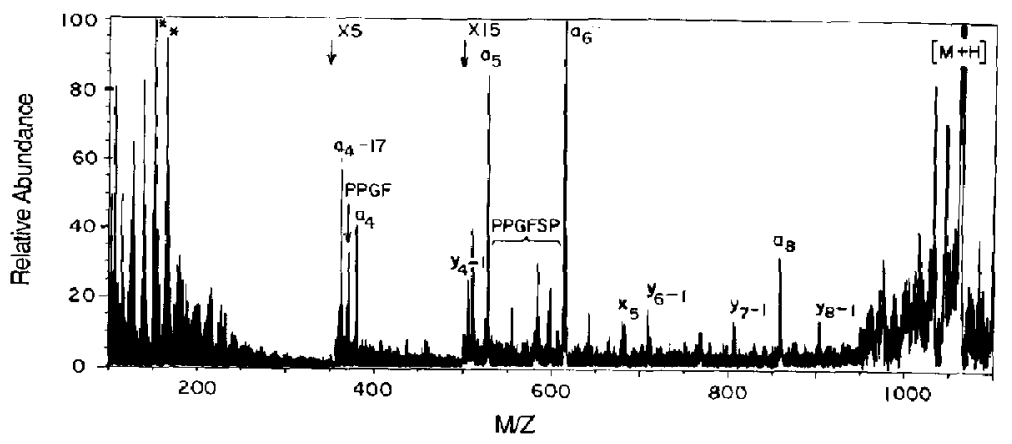

Figure 2. Product ions formed by losses of 28 $\mathrm{u}$ in the fragmentation of bradykinin (Arg-ProPro-Gly-Phe-Ser-Pro-Phe-Arg), monoisotopic mass $1059.6 \mathrm{u}$, analyzed by CNL scan. Cations were desorbed by FAB from an NBA matrix. 
Figure 3. Product ions formed by loss of $28 \mathrm{u}$ in the fragmentation of porcine renin substrate tetradecapeptide (Asp-Arg-Val-Tyr-Ile-His-Pro-Phe-His-Leu-Leu-ValTyr Ser), monoisotropic mass $1757.9 \mathrm{u}$, analyzed by CNL scan. Cations were desorbed by FAB from a thioglycerol matrix. Thioglycerol matrix cluster ions are indicated by

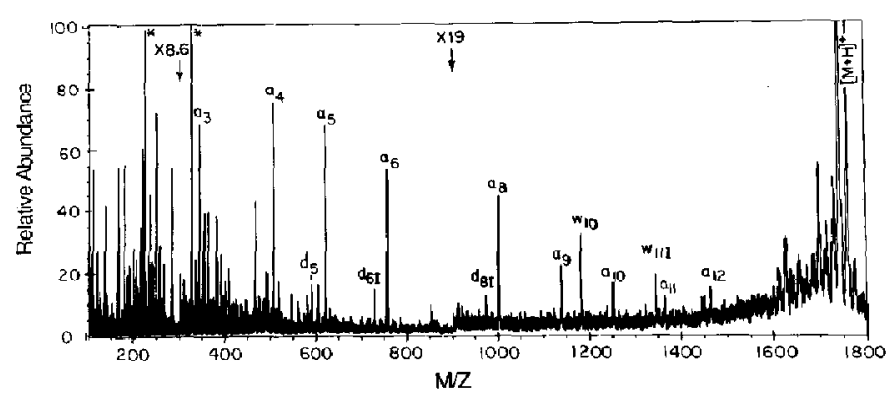

abundant $b_{5}$ ion is unusual. However, the occurrence of glycine as residue 6 means that $a_{6}$ may be the precursor.<smiles>[R3]C(C=[O+])NCCNC(=O)C([R3])N</smiles>

Arginine in position 8 probably carries the charge that allows detection of members of the $x$ series, $x_{3}-x_{5}$ and $x_{9}$. Ions $x_{4}$ and $x_{9}$ are probably formed from $v_{5}$ and $w_{10}$ ions, respectively, since the alternate precursors $w_{5}$ and $v_{10}$ cannot be formed from glycine and pyroglutamate, respectively. Doubly charged $[\mathrm{M}+2 \mathrm{H}]^{2+}$ ions detected in the conventional magnet $\operatorname{scan}(m / z$ 591) contribute ions at $m / z 563$ in the CNL scan by loss of 56 and/or $57 \mathrm{u}$. Singly charged $[\mathrm{MH}-56]^{+}$and
$[\mathrm{MH}-57]^{+}$ions are detected in the conventional scan (Figure 1b).

The CNL 28 u spectrum obtained from bradykinin is presented in Figure 2. This nonapeptide carries an arginine at each end, and sequence ions (a and $y-1$ series) are detected with comparable abundances from both ends in the CNL 28 u spectrum, the four-sector product ion spectrum, and the conventional scan. The conventional and four-sector product ion spectra both contain $[\mathrm{MH}-86]^{+}$and $[\mathrm{MH}-100]^{+}$ions formed by cleavage in the arginine side chain. In the CNL $28 \mathrm{u}$ spectrum, ions $a_{4}-a_{6}$ and $a_{8}$ are detected. Ions $y_{4}-1$ and $y_{6}-1$ through $y_{8}-1$ are also detected, consistent with the presence of the precursor $x+1$ series in the conventional magnet scan. (The occurrence of prolines as the $\mathrm{N}$-terminus residues in the $y_{7}-1$ and $y_{8}-1$ ions is accommodated by minor modification of the $y_{n}-1$ structure in the general scheme drawn above). Presumably the extra proton is carried on C-terminal arginine, and neutral $\mathrm{CO}$ is eliminated as a remote site $[5,10]$
Figure 4. Product cations formed by loss of $28 \mathrm{u}$ in the fragmentation of (a) $p$ nitrobenzyl alcohol-0.1\% TFA $1: 1$ and (b) thioglycerol- $0.1 \%$ trifluoroacetic acid 1:1

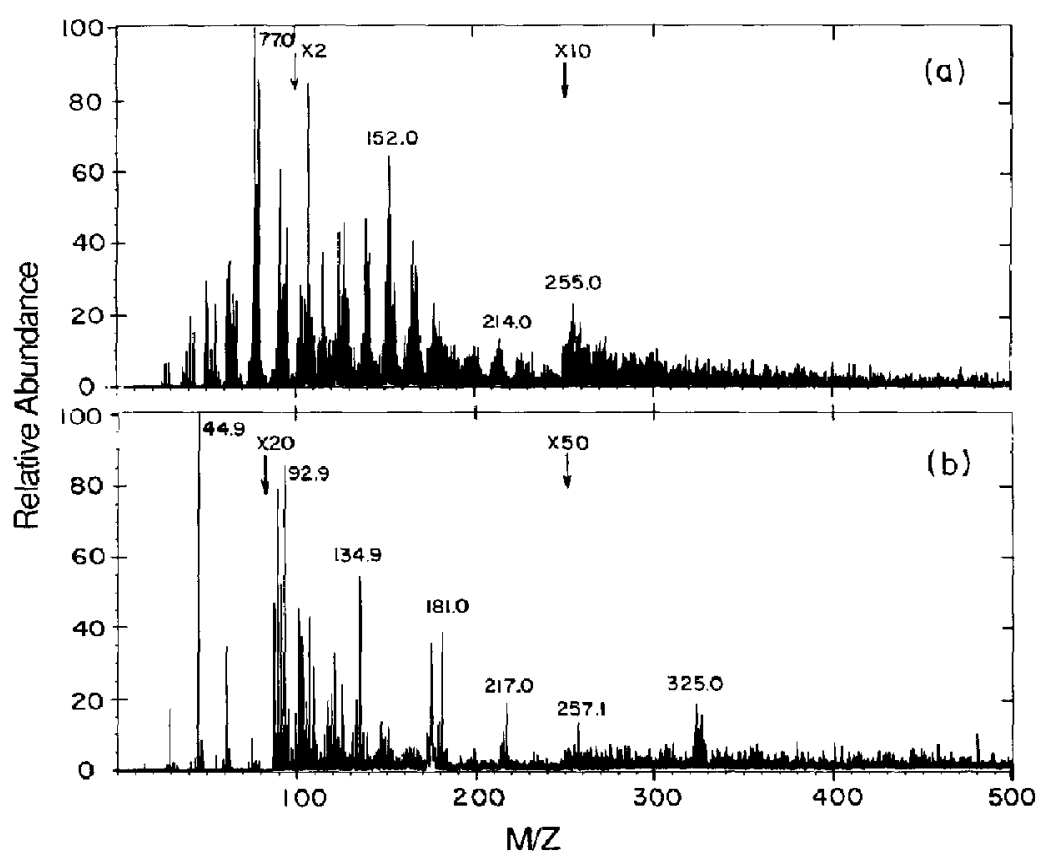


fragmentation. Ions of masses $371,555,584$, and 598 $\mathbf{u}$ are interpreted as pieces of an internal peptide fragment PPGFSP. Below $m / z 250$ the spectrum resembles the NBA spectrum in Figure 4.

The CNL 28 spectrum of renin substrate is shown in Figure 3. Like the tandem spectrum and the conventional magnet scan, it is dominated by ion series that include the arginine residue near the amino terminus. In the conventional magnet scan, additional carboxy terminus ions are present, and in the four-sector product ion scan a number of $d$ ions are identified [9]. In the case of an unknown sample, the commonality of the a series in the CNL $28 \mathrm{u}$ and four-sector product ion spectra might facilitate interpretation. Ions formed by side-chain cleavage observed in the CNL $28 \mathrm{u}$ and four-sector product ion spectra differ and provide complementary information. Of particular interest in the CNL $28 \mathrm{u}$ spectrum is the contribution of isoleucine (residue 5). The loss of $\mathrm{C}_{2} \mathrm{H}_{4}$ from the side chain has been remarked by both Johnson et al. [11] and Carr et al. [12] and here contributes $d_{5}, d_{61}, d_{81}, w_{10}$, and $w_{111}$ ions. The clarity of this spectrum of a $1750-u$ peptide augurs well for the use of CNL scanning through the mass range to $2000 \mathrm{u}$.

\section{Conclusions}

The CNL spectra recorded in this study generally have $S / N$ ratios superior to those of conventional magnet $B^{2}$ scans but not as favorable as those in product ion $(B / E)$ scans in MS-2 following collisional activation in the four-sector instrument. Often the CNL scans contain fewer fragment ions than the four-sector product ion spectra. Fewer side-chain cleavages are detected, and the ion series found (a versus $x$ and $y-1$ ) reflect the positions of basic amino acids in the chain. Theory predicts and experiments confirm that ions detected in the CNL $28 \mathrm{u}$ spectra have only a limited number of origins. The ions most often detected in this manner in the present study were series a sequence ions. In the fortuitous case, these can provide the entire sequence. In the more general case, the CNL $28 \mathrm{u}$ spectrum can be used as a reading frame to recognize, by either omission or coincidence, ion series in product ion spectra produced by collisional activation in tandem mass spectrometry experiments.

\section{Acknowledgment}

The studies reported here were supported by grants BBS 8714238 and DMB 8610589 from the National Science Foundation.

\section{References}

1. Lacey, M. J.; Macdonald, C. G. Atral. Chem. 1979, 51, 691-695.

2. Zackett, D.; Schoen, A. E.; Kondrat, R. W.; Cooks, R. G. J. Am. Chem. Soc. 1979, 101, 6781-6783.

3. Haddon, W. F. Org. Mass Spectrom. 1980, 15, 539-543.

4. DePauw, E.; Pelzer, G.; Nasar, K. Biomed. Environ. Mass Spectrom. 1988, 15, 577-581.

5. Johnson, R. S.; Martin, S. A.; Biemann, K. Int. I. Mass Spectrom. Inn Prorrsses 1988, 86, 137-154

6. Hunt, D. F.; Shabanowitz, J.; Yates, J. R. J. Chem. Soc., Chem. Commun. 1987, 548-549.

7. Orlando, R.; Murphy, C.; Fenselau, C.; Hansen, G. E.; Cotter, R. J. Anal. Chem. 1990, 62, 125-129.

8. Orlando, R.; Fenselau, C.; Cotter, R. J. Organic Mass Spectrom. 1989, 24, 1033-1042.

9. Martin, S. A.; Johnson, R. S.; Costello, C. E.; Biemann, K. In The Analysis of Peptides and Proteins by Mass Spectrometry: McNeal, C. J., Ed.; Wiley: New York, 1988; pp 135-150.

10. Jensen, N. J.; Tomer, K. B.; Gross, M. L. J. Am. Chem. Sac. $1985,107,1863-1866$.

11. Johnson, R. S.; Martin, S. A.; Biemann, K.; Stults, J. T.; Watson, J. T. Anal. Chem. 1987, 59, 2621.

12. Carr, 5. A.; Green, B. N.; Hemling, M. E.; Roberts, G. U.; Anderegg, R. J.; Vickers, R. Proceedings of the 35th Annual ASMS Conference on Mass Spectrometry and Allied Topics; Denver, 1987, p 830. 